\title{
SOME REMARKS ABOUT STIRLING NUMBERS OF THE SECOND KIND
}

\section{Ramiz Vugdalić ${ }^{1}$ \\ Fatih Destović}

Faculty of Science, University of Tuzla

Faculty of Educational Sciences, University of Sarajevo

Received: 12.03 .2013 .

Accepted: 15.04.2013.
Professional paper

UDC: 511.178

\begin{abstract}
In this paper we give a representation of Stirling numbers of the second kind, we obtain explicit formulas for some cases of Stirling numbers of the second kind and illustrate a method for founding other such formulas.

2010 Mathematics Subject Classification. 11B73, 05A10.
\end{abstract}

Key words: Combinatorics sum, Stirling numbers of the second kind.

\section{PRELIMINARIES}

In Combinatorial Theory (Aigner, 1979) are given the properties and the values of combinatorics sum

$S(n, l):=\sum_{k=0}^{n}(-1)^{k}\left(\begin{array}{l}n \\ k\end{array}\right)(n-k)^{l} \quad(n, l \in \mathrm{N} \cup\{0\})$.

Especially, we define that $\mathrm{S}(0,0):=1$. It is a known fact that $S(n, n)=n$ ! for every $n \in N$, and $S(n, 1)=0$ for $1<\mathrm{n}$ : For $\mathrm{n}<1$; the sum $\mathrm{S}(\mathrm{n}, \mathrm{l})$ is interesting because in combinatorics it represents the number of all possible permutations with repetitions of a set of 1 elements with $n$ classes ([2], page 216), i.e., for $\mathrm{n}<1$;

$$
S(n, l)=\sum_{\substack{k_{1}, \ldots, k_{n} \in \mathrm{N} \\ k_{1}+\ldots+k_{n}=l}} \frac{l !}{k_{1} ! \ldots k_{n} !}=l ! \sum_{\substack{k_{1}, \ldots, k_{n} \in \mathrm{N} \\ k_{1}+\ldots+k_{n}=l}} \frac{1}{k_{1} ! \ldots k_{n} !}
$$

\footnotetext{
${ }^{1}$ Correspondence to:

Ramiz Vugdalić, Faculty of Science, University of Tuzla

E-mail: ramiz.vugdalic@untz.ba
}

Also, there exists a relationship for the sum S(n,1) using Stirling numbers of the second kind. Namely, for

$$
S(n, l)=n ! \cdot S_{2}(l, n)
$$

where with S2(1,n) we denote Stirling numbers of the second kind (Velja, 2001, pp. 100-101). The Stirling numbers of the second kind S2 $(1, n)$ describe the number of ways a set with 1 elements can be partitioned into $\mathrm{n}$ disjoint non-empty subsets. In Combinatorial Theory (Aigner, 1979) it is proved the following representation of $\mathrm{S}(\mathrm{n}, 1)$ for $\mathrm{n}<1$.

Theorem 1 (Theorem 7. in Combinatorial Theory (Aigner, 1979) For every $k \in N$ there exists a polynomial $f_{k}(n)=a_{1} n^{k}+\ldots+a_{k} n$ with rational coefficients and with property that for every $n \in \mathrm{N} \cup\{0\}$

$S(n, n+k)=f_{k}(n)(n+k) !$

Also, the following holds 


$$
\sum_{i=1}^{k} a_{i}=\frac{1}{(k+1) !}
$$

Especially, for every $n \in \mathrm{N} \cup\{0\}$ are obtained the following concrete formulas (formulas (7)-(11) in [1]):

$$
\begin{aligned}
& S(n, n+1)=\frac{n}{2}(n+1) ! \\
& S(n, n+2)=\frac{3 n^{2}+n}{24}(n+2) ! \\
& S(n, n+3)=\frac{n^{3}+n^{2}}{48}(n+3) ! \\
& S(n, n+4)=\frac{15 n^{4}+30 n^{3}+5 n^{2}-2 n}{5760}(n+4) ! \\
& S(n, n+5)=\frac{9 n^{5}+30 n^{4}+15 n^{3}-6 n^{2}}{34560}(n+5) !
\end{aligned}
$$

These formulas hold for every $n \in \mathrm{N} \cup\{0\}$. Therefore, the coefficients of the polynomial $f_{k}(n)$ depend only of $\mathrm{k}$.

\section{RESULTS}

From (1) and (2) we conclude that Stirling numbers of the second kind $S_{2}(n+k, n)$ have the following representation:

$S_{2}(n+k, n)=\frac{(n+k) !}{n !} \cdot f_{k}(n)=\left(\begin{array}{c}n+k \\ k\end{array}\right) \cdot g_{k}(n)$

with $g_{k}(n):=k ! \cdot f_{k}(n)$ for $n, k \in \mathrm{N} \cup\{0\}$. We want to see how the coefficients of polynomials $\mathrm{f}_{\mathrm{k}}(\mathrm{n})$ and $\mathrm{g}_{\mathrm{k}}(\mathrm{n})$ depend of $\mathrm{k}$ : From theorem 2. in Combinatorial Theory (Aigner, 1979) we have the recurrence relation:

$S(n, n+k)=n \cdot S(n-1, n+k-1)+n \cdot S(n, n+k-1)$

Using (2) this means

$$
f_{k}(n) \cdot(n+k) !=n \cdot f_{k}(n-1) \cdot(n-1+k) !+n \cdot f_{k-1}(n) \cdot(n+k-1) !
$$

Now, if we divide the last relation with $(n+k-1)$ !, then we obtain the equality

$$
f_{k}(n) \cdot(n+k)=n \cdot f_{k}(n-1)+n \cdot f_{k-1}(n)
$$

Denote :

$$
f_{k}(n)=a_{1}(k) n^{k}+a_{2}(k) n^{k-1}+\ldots+a_{k}(k) n
$$

Then, from (10) we have further

$$
\begin{gathered}
(n+k) \cdot\left[a_{1}(k) n^{k}+a_{2}(k) n^{k-1}+\ldots+a_{k}(k) n\right]= \\
=n \cdot\left[a_{1}(k)(n-1)^{k}+a_{2}(k)(n-1)^{k-1}+\ldots+a_{k}(k)(n-1)\right] \\
+n \cdot\left[a_{1}(k-1) n^{k-1}+a_{2}(k-1) n^{k-2}+\ldots+a_{k-1}(k-1) n\right]
\end{gathered}
$$

Observing the coefficients by $\mathrm{n}^{\mathrm{k}}$ in (11) we have

$$
\begin{array}{r}
\text { i.e., } \quad k \cdot a_{1}(k)+a_{2}(k)=-k \cdot a_{1}(k)+a_{2}(k)+a_{1}(k-1), \\
a_{1}(k)=\frac{1}{2 k} \cdot a_{1}(k-1) \quad(*)
\end{array}
$$

Because of ${ }_{a 1}(0)=1$, we conclude that

$$
a_{1}(k)=\frac{1}{2^{k} \cdot k !} \quad(k \in \mathrm{N} \cup\{0\})
$$

Now, we observe the coefficients by $\mathrm{n}^{\mathrm{k}-1}$ in (11), and we get

$$
\begin{aligned}
& k \cdot a_{2}(k)+a_{3}(k)=\left(\begin{array}{l}
k \\
2
\end{array}\right) a_{1}(k)-\left(\begin{array}{c}
k-1 \\
1
\end{array}\right) a_{2}(k)+a_{3}(k)+a_{2}(k-1), \\
& \text { i.e., } \\
& (2 k-1) a_{2}(k)=\left(\begin{array}{l}
k \\
2
\end{array}\right) a_{1}(k)+a_{2}(k-1) .
\end{aligned}
$$

Using (12) we have further,

$$
(2 k-1) a_{2}(k)=a_{2}(k-1)+\frac{\left(\begin{array}{l}
k \\
2
\end{array}\right)}{2^{k} \cdot k !}
$$

By mathematical induction we want to prove that

$$
a_{2}(k)=\frac{\frac{1}{3}\left(\begin{array}{l}
k \\
2
\end{array}\right)}{2^{k} \cdot k !} \quad(k \in \mathrm{N} \cup\{0\})
$$
Because of $a_{2}(0)=a_{2}(1)=0$ and $a_{2}(2)=\frac{1}{24}$, we
conclude that $(13)$ holds for $\mathrm{k}=1,2$.

If we assume that

$$
a_{2}(k-1)=\frac{\frac{1}{3}\left(\begin{array}{c}
k-1 \\
2
\end{array}\right)}{2^{k-1} \cdot(k-1)}
$$


then from $(* *)$ we have

$$
\begin{aligned}
(2 k-1) a_{2}(k) & =\frac{\frac{1}{3}\left(\begin{array}{c}
k-1 \\
2
\end{array}\right)}{2^{k-1} \cdot k-1 !}+\frac{\left(\begin{array}{l}
k \\
2
\end{array}\right)}{2^{k} \cdot k !}=\frac{\frac{2 k}{3}\left(\begin{array}{c}
k-1 \\
2
\end{array}\right)+\left(\begin{array}{l}
k \\
2
\end{array}\right)}{2^{k} \cdot k !}= \\
& =\frac{\left(\begin{array}{l}
k \\
2
\end{array}\right)}{2^{k} \cdot k !} \cdot\left[1+\frac{2}{3}(k-2)\right]=\frac{\left(\begin{array}{l}
k \\
2
\end{array}\right)}{2^{k} \cdot k !} \cdot \frac{2 k-1}{3} .
\end{aligned}
$$

Hence, (13) holds. Observe now the coefficients by $\mathrm{n}^{\mathrm{k}-2}$ in (11). Then, we have

$$
k \cdot a_{3}(k)+a_{4}(k)=-\left(\begin{array}{l}
k \\
3
\end{array}\right) a_{1}(k)+\left(\begin{array}{c}
k-1 \\
2
\end{array}\right) a_{2}(k)-\left(\begin{array}{c}
k-2 \\
2
\end{array}\right) a_{3}(k)+a_{4}(k)+a_{3}(k-1)
$$

Hence,

$$
(2 k-2) a_{3}(k)=a_{3}(k-1)-\left(\begin{array}{l}
k \\
3
\end{array}\right) \cdot \frac{1}{2^{k} \cdot k !}+\left(\begin{array}{c}
k-1 \\
2
\end{array}\right) \cdot \frac{\frac{1}{3}\left(\begin{array}{l}
k \\
2
\end{array}\right)}{2^{k} \cdot k !}
$$
i.e.,

$$
(2 k-2) a_{3}(k)=a_{3}(k-1)+\frac{2\left(\begin{array}{l}
k \\
4
\end{array}\right)}{2^{k} \cdot k !}
$$

By mathematical induction we want to prove that

$$
\left.a_{3}(k)=\frac{\frac{1}{3}\left(\begin{array}{l}
k \\
4
\end{array}\right)}{2^{k} \cdot k !} \quad k \in \mathrm{N} \cup\{0\}\right)
$$

It is clear that $a_{3}(k)=0$ for $k \leq 3$, i.e. (14) holds for $k \leq 3$. If we assume that

$$
a_{3}(k-1)=\frac{\frac{1}{3}\left(\begin{array}{c}
k-1 \\
4
\end{array}\right)}{2^{k-1} \cdot(k-1) !}
$$

then from $(* * *)$ we have

$$
\begin{aligned}
(2 k-2) a_{3}(k) & =\frac{\frac{1}{3}\left(\begin{array}{c}
k-1 \\
4
\end{array}\right)}{2^{k-1} \cdot(k-1) !}+\frac{2\left(\begin{array}{l}
k \\
4
\end{array}\right)}{2^{k} \cdot k !}=\frac{\frac{2 k}{3}\left(\begin{array}{c}
k-1 \\
4
\end{array}\right)+2\left(\begin{array}{l}
k \\
4
\end{array}\right)}{2^{k} \cdot k !}= \\
& =\frac{\left(\begin{array}{l}
k \\
4
\end{array}\right)}{2^{k} \cdot k !} \cdot\left[2+\frac{2}{3}(k-4)\right]=\frac{\left(\begin{array}{l}
k \\
4
\end{array}\right)}{2^{k} \cdot k !} \cdot \frac{2 k-2}{3} .
\end{aligned}
$$

Hence, (14) holds. Note that formulas (13) and (14) also we can obtain using the substitution
$a_{2}(k)=\frac{A\left(\begin{array}{l}k \\ 2\end{array}\right)}{2^{k} \cdot k !}, \quad a_{3}(k)=\frac{B\left(\begin{array}{l}k \\ 4\end{array}\right)}{2^{k} \cdot k !} \quad(k \in \mathrm{N} \cup\{0\})$

in $(* *)$ and $(* * *)$ and finding $A=B=\frac{1}{3}$. Observe now the coefficients by $\mathrm{n}^{\mathrm{k}-3}$ in (11). Then, we have

$$
\begin{aligned}
& k \cdot a_{4}(k)+a_{5}(k)=\left(\begin{array}{l}
k \\
4
\end{array}\right) a_{1}(k)-\left(\begin{array}{c}
k-1 \\
3
\end{array}\right) a_{2}(k)+\left(\begin{array}{c}
k-2 \\
2
\end{array}\right) a_{2}(k) \\
& -\left(\begin{array}{c}
k-3 \\
1
\end{array}\right) a_{4}(k)+a_{4}(k-1)+a_{5}(k), \\
& (2 k-3) a_{4}(k)=a_{4}(k-1)+\frac{1}{2^{k} \cdot k !}\left[\left(\begin{array}{l}
k \\
4
\end{array}\right)-\frac{1}{3}\left(\begin{array}{l}
k \\
2
\end{array}\right)\left(\begin{array}{c}
k-1 \\
3
\end{array}\right)+\frac{1}{3}\left(\begin{array}{c}
k \\
4
\end{array}\right)\left(\begin{array}{c}
k-2 \\
2
\end{array}\right)\right] .
\end{aligned}
$$

Since

$$
\begin{gathered}
\left(\begin{array}{l}
k \\
4
\end{array}\right)-\frac{1}{3}\left(\begin{array}{l}
k \\
2
\end{array}\right)\left(\begin{array}{c}
k-1 \\
3
\end{array}\right)+\frac{1}{3}\left(\begin{array}{l}
k \\
4
\end{array}\right)\left(\begin{array}{c}
k-2 \\
2
\end{array}\right)= \\
=\left(\begin{array}{l}
k \\
4
\end{array}\right) \cdot\left[1-\frac{2}{3}(k-1)+\frac{1}{6}(k-2)(k-3)\right] \\
=\left(\begin{array}{l}
k \\
4
\end{array}\right) \cdot \frac{1}{6}\left(k^{2}-9 k+16\right)=\frac{1}{6}\left(\begin{array}{l}
k \\
4
\end{array}\right) \cdot[(k-4)(k-5)-4] \\
=5\left(\begin{array}{l}
k \\
6
\end{array}\right)-\frac{2}{3}\left(\begin{array}{l}
k \\
4
\end{array}\right),
\end{gathered}
$$

we have the recurrence relation

$$
(2 k-3) a_{4}(k)=a_{4}(k-1)+\frac{5\left(\begin{array}{l}
k \\
6
\end{array}\right)-\frac{2}{3}\left(\begin{array}{l}
k \\
4
\end{array}\right)}{2^{k} \cdot k !}
$$

Look for the solution of the last equation in the form

$$
a_{4}(k)=\frac{A\left(\begin{array}{l}
k \\
6
\end{array}\right)+B\left(\begin{array}{l}
k \\
4
\end{array}\right)}{2^{k} \cdot k !}
$$

for some rational constants $\mathrm{A}$ and $\mathrm{B}$ : Then, from $(* * * *)$ we have

$$
(2 k-3) \cdot\left[A\left(\begin{array}{l}
k \\
6
\end{array}\right)+B\left(\begin{array}{l}
k \\
4
\end{array}\right)\right]=2 k \cdot\left[A\left(\begin{array}{c}
k-1 \\
6
\end{array}\right)+B\left(\begin{array}{c}
k-1 \\
4
\end{array}\right)\right]+5\left(\begin{array}{l}
k \\
6
\end{array}\right)-\frac{2}{3}\left(\begin{array}{l}
k \\
4
\end{array}\right) .
$$

Fruther,

$$
(2 k-3) \cdot A\left(\begin{array}{l}
k \\
6
\end{array}\right)=2 k \cdot A\left(\begin{array}{c}
k-1 \\
6
\end{array}\right)+5\left(\begin{array}{l}
k \\
6
\end{array}\right)
$$


implies $\quad(2 k-3) \cdot A=2(k-6) \cdot A+5$

Hence, $A=\frac{5}{9}$. Also, from

$$
(2 k-3) \cdot B\left(\begin{array}{l}
k \\
4
\end{array}\right)=2 k \cdot B\left(\begin{array}{c}
k-1 \\
4
\end{array}\right)-\frac{2}{3}\left(\begin{array}{l}
k \\
4
\end{array}\right)
$$

we obtain

$$
(2 k-3) \cdot B=2(k-4) \cdot B-\frac{2}{3}
$$

Therefore, $B=-\frac{2}{15}$. Hence,

$a_{4}(k)=\frac{\frac{5}{9}\left(\begin{array}{l}k \\ 6\end{array}\right)-\frac{2}{15}\left(\begin{array}{l}k \\ 4\end{array}\right)}{2^{k} \cdot k !} \quad(k \in \mathrm{N} \cup\{0\})$

Observe now the coefficients by $\mathrm{n}^{\mathrm{k}-4}$ in (11). Then, we have

$$
\begin{gathered}
k \cdot a_{5}(k)+a_{6}(k)=-\left(\begin{array}{c}
k \\
5
\end{array}\right) a_{1}(k)+\left(\begin{array}{c}
k-1 \\
4
\end{array}\right) a_{2}(k)-\left(\begin{array}{c}
k-2 \\
3
\end{array}\right) a_{3}(k) \\
+\left(\begin{array}{c}
k-3 \\
2
\end{array}\right) a_{4}(k)-\left(\begin{array}{c}
k-4 \\
1
\end{array}\right) a_{5}(k)+a_{5}(k-1)+a_{6}(k),
\end{gathered}
$$

i.e.,

$$
\begin{gathered}
(2 k-4) a_{5}(k)=a_{5}(k-1)+\frac{1}{2^{k} \cdot k !}\left[-\left(\begin{array}{l}
k \\
5
\end{array}\right)+\frac{1}{3}\left(\begin{array}{l}
k \\
2
\end{array}\right)\left(\begin{array}{c}
k-1 \\
4
\end{array}\right)\right] \\
-\frac{1}{3}\left(\begin{array}{l}
k \\
4
\end{array}\right)\left(\begin{array}{c}
k-2 \\
3
\end{array}\right)+\left(\begin{array}{c}
k-3 \\
2
\end{array}\right)\left(\frac{5}{9}\left(\begin{array}{l}
k \\
6
\end{array}\right)-\frac{2}{15}\left(\begin{array}{l}
k \\
4
\end{array}\right)\right) .
\end{gathered}
$$

Because

$$
\begin{gathered}
-\left(\begin{array}{l}
k \\
5
\end{array}\right)+\frac{1}{3}\left(\begin{array}{l}
k \\
2
\end{array}\right)\left(\begin{array}{c}
k-1 \\
4
\end{array}\right)-\frac{1}{3}\left(\begin{array}{l}
k \\
4
\end{array}\right)\left(\begin{array}{c}
k-2 \\
3
\end{array}\right)+\left(\begin{array}{c}
k-2 \\
3
\end{array}\right)\left(\frac{5}{9}\left(\begin{array}{l}
k \\
6
\end{array}\right)-\frac{2}{15}\left(\begin{array}{l}
k \\
4
\end{array}\right)\right)= \\
=\left(\begin{array}{l}
k \\
5
\end{array}\right) \cdot \frac{1}{108}\left(5 k^{3}-90 k^{2}+439 k-570\right) \\
=\left(\begin{array}{l}
k \\
5
\end{array}\right) \cdot \frac{1}{108} \cdot[5(k-5)(k-6)(k-7)-96(k-5)] \\
=\frac{140}{9}\left(\begin{array}{l}
k \\
8
\end{array}\right)-\frac{48}{9}\left(\begin{array}{l}
k \\
6
\end{array}\right)
\end{gathered}
$$

Hence, $A=\frac{35}{27}$. Also, from

$$
(2 k-4) \cdot B\left(\begin{array}{l}
k \\
6
\end{array}\right)=2 k \cdot B\left(\begin{array}{c}
k-1 \\
6
\end{array}\right)-\frac{48}{9}\left(\begin{array}{l}
k \\
6
\end{array}\right)
$$

we obtain

$$
(2 k-4) \cdot B=2(k-6) \cdot B-\frac{48}{9} .
$$

Therefore, $B=-\frac{2}{3}$. Hence,

we have the recurrence relation 
$a_{5}(k)=\frac{\frac{35}{27}\left(\begin{array}{l}k \\ 8\end{array}\right)-\frac{2}{3}\left(\begin{array}{l}k \\ 6\end{array}\right)}{2^{k} \cdot k !} \quad(k \in \mathrm{N} \cup\{0\})$

Observing the coefficients by $\mathrm{n}^{\mathrm{k}-5}$ in (11) we obtain $(2 k-5) a_{6}(k)=a_{6}(k-1)+\frac{1}{2^{k} \cdot k !} \cdot\left[\left(\begin{array}{l}k \\ 6\end{array}\right)-\frac{1}{3}\left(\begin{array}{l}k \\ 2\end{array}\right)\left(\begin{array}{c}k-1 \\ 5\end{array}\right)+\frac{1}{3}\left(\begin{array}{l}k \\ 4\end{array}\right)\left(\begin{array}{c}k-2 \\ 4\end{array}\right)\right]$
$-\left(\begin{array}{c}k-3 \\ 3\end{array}\right)\left(\frac{5}{9}\left(\begin{array}{l}k \\ 6\end{array}\right)-\frac{2}{15}\left(\begin{array}{l}k \\ 4\end{array}\right)\right)+\left(\begin{array}{c}k-4 \\ 2\end{array}\right)\left(\frac{35}{27}\left(\begin{array}{l}k \\ 8\end{array}\right)-\frac{2}{3}\left(\begin{array}{l}k \\ 6\end{array}\right)\right)$

Because

$$
\begin{gathered}
\left(\begin{array}{l}
k \\
6
\end{array}\right)-\frac{1}{3}\left(\begin{array}{l}
k \\
2
\end{array}\right)\left(\begin{array}{c}
k-1 \\
5
\end{array}\right)+\frac{1}{3}\left(\begin{array}{l}
k \\
4
\end{array}\right)\left(\begin{array}{c}
k-2 \\
4
\end{array}\right)-\left(\begin{array}{c}
k-3 \\
3
\end{array}\right)\left(\frac{5}{9}\left(\begin{array}{l}
k \\
6
\end{array}\right)-\frac{2}{15}\left(\begin{array}{l}
k \\
4
\end{array}\right)\right) \\
+\left(\begin{array}{c}
k-4 \\
2
\end{array}\right)\left(\frac{35}{27}\left(\begin{array}{l}
k \\
8
\end{array}\right)-\frac{2}{3}\left(\begin{array}{l}
k \\
6
\end{array}\right)\right)=
\end{gathered}
$$

$=\left(\begin{array}{l}k \\ 6\end{array}\right) \cdot \frac{1}{432} \cdot\left[5 k^{4}-150 k^{3}+1411 k^{2}-4818 k+4800\right]$

$=\left(\begin{array}{l}k \\ 6\end{array}\right) \cdot \frac{1}{432} \cdot[5(k-6)(k-7)(k-8)(k-9)-264(k-6)(k-7)+768]$ $=\frac{175}{3}\left(\begin{array}{c}k \\ 10\end{array}\right)-\frac{308}{9}\left(\begin{array}{l}k \\ 8\end{array}\right)+\frac{768}{432}\left(\begin{array}{l}k \\ 6\end{array}\right)$

we have the recurrence relation

$(2 k-5) a_{6}(k)=a_{6}(k-1)+\frac{\frac{175}{3}\left(\begin{array}{c}k \\ 10\end{array}\right)-\frac{308}{9}\left(\begin{array}{l}k \\ 8\end{array}\right)+\frac{768}{432}\left(\begin{array}{l}k \\ 6\end{array}\right)}{2^{k} \cdot k !}$

$(* * * * * *)$

If we search the solution of the last equation in the form

$$
a_{6}(k)=\frac{A\left(\begin{array}{c}
k \\
10
\end{array}\right)+B\left(\begin{array}{l}
k \\
8
\end{array}\right)+C\left(\begin{array}{l}
k \\
6
\end{array}\right)}{2^{k} \cdot k !}
$$

for some rational constants $\mathrm{A}, \mathrm{B}$ and $\mathrm{C}$; then, from $(* * * * *)$ we have

$$
\begin{gathered}
(2 k-5) \cdot\left[A\left(\begin{array}{c}
k \\
10
\end{array}\right)+B\left(\begin{array}{l}
k \\
8
\end{array}\right)+C\left(\begin{array}{l}
k \\
6
\end{array}\right)\right]= \\
=2 k \cdot\left[A\left(\begin{array}{c}
k-1 \\
10
\end{array}\right)+B\left(\begin{array}{c}
k-1 \\
8
\end{array}\right)+C\left(\begin{array}{c}
k-1 \\
6
\end{array}\right)\right]+\frac{175}{3}\left(\begin{array}{c}
k \\
10
\end{array}\right)-\frac{308}{9}\left(\begin{array}{l}
k \\
8
\end{array}\right)+\frac{768}{432}\left(\begin{array}{l}
k \\
6
\end{array}\right) .
\end{gathered}
$$

Solving this equation, we obtain :

$$
A=\frac{35}{9}, \quad B=-\frac{28}{9}, \quad C=\frac{16}{63} .
$$

Hence,

$a_{6}(k)=\frac{\frac{35}{9}\left(\begin{array}{c}k \\ 10\end{array}\right)-\frac{28}{9}\left(\begin{array}{l}k \\ 8\end{array}\right)+\frac{16}{63}\left(\begin{array}{l}k \\ 6\end{array}\right)}{2^{k} \cdot k !} \quad(k \in \mathrm{N} \cup\{0\})$

If we continue the described method, we can obtain explicit formulas for coefficients ${ }_{a 7}(\mathrm{k}){ }_{\mathrm{a} 8}(\mathrm{k})$ and other coefficients of the polynomial $\mathrm{f}_{\mathrm{k}}(\mathrm{n})$. Finally, using Theorem 1, formulas (9), (12)-(17), we have proved the following theorem.

Theorem 2 For every $k \in \mathrm{N} \cup\{0\}$ there exists a unique polynomial $g_{k}(n)=b_{1}(k) n^{k}+b_{2}(k) n^{k-1}+\ldots+b_{k}(k) n$ with rational coefficients and with property that for every $n \in \mathrm{N} \cup\{0\}$,

$$
S_{2}(n+k, n)=\left(\begin{array}{c}
n+k \\
k
\end{array}\right) g_{k}(n)
$$

Also, the following holds

$$
\sum_{i=1}^{k} b_{i}(k)=\frac{1}{k+1} .
$$

The first six coefficients of the polynomial $g_{k}(n)$ are equal:

$b_{1}(k)=\frac{1}{2^{k}}, \quad b_{2}(k)=\frac{\frac{1}{3}\left(\begin{array}{l}k \\ 2\end{array}\right)}{2^{k}}, \quad b_{3}(k)=\frac{\frac{1}{3}\left(\begin{array}{l}k \\ 4\end{array}\right)}{2^{k}}, \quad b_{4}(k)=\frac{\frac{5}{9}\left(\begin{array}{l}k \\ 6\end{array}\right)-\frac{2}{15}\left(\begin{array}{l}k \\ 4\end{array}\right)}{2^{k}}$,
$b_{5}(k)=\frac{\frac{35}{27}\left(\begin{array}{l}k \\ 8\end{array}\right)-\frac{2}{3}\left(\begin{array}{l}k \\ 6\end{array}\right)}{2^{k}}, \quad b_{6}(k)=\frac{\frac{35}{9}\left(\begin{array}{c}k \\ 10\end{array}\right)-\frac{28}{9}\left(\begin{array}{l}k \\ 8\end{array}\right)+\frac{16}{63}\left(\begin{array}{l}k \\ 6\end{array}\right)}{2^{k}} \quad(k \in \mathrm{N} \cup\{0\})$.

Corollary 3 For every $n \in \mathrm{N} \cup\{0\}$ the following formulas hold:

$$
\begin{aligned}
& S_{2}(n+1, n)=\left(\begin{array}{c}
n+1 \\
1
\end{array}\right) \cdot \frac{n}{2} \\
& S_{2}(n+2, n)=\left(\begin{array}{c}
n+2 \\
2
\end{array}\right) \cdot \frac{3 n^{2}+n}{12} \\
& S_{2}(n+3, n)=\left(\begin{array}{c}
n+3 \\
3
\end{array}\right) \cdot \frac{n^{3}+n^{2}}{8} \\
& S_{2}(n+4, n)=\left(\begin{array}{c}
n+4 \\
4
\end{array}\right) \cdot \frac{15 n^{4}+30 n^{3}+5 n^{2}-2 n}{240} \\
& S_{2}(n+5, n)=\left(\begin{array}{c}
n+5 \\
5
\end{array}\right) \cdot \frac{9 n^{5}+30 n^{4}+15 n^{3}-6 n^{2}}{288} \\
& S_{2}(n+6, n)=\left(\begin{array}{c}
n+6 \\
6
\end{array}\right) \cdot \frac{63 n^{6}+315 n^{5}+315 n^{4}-91 n^{3}-42 n^{2}+16 n}{4032}
\end{aligned}
$$

Remark 4 Note that the formulas (18)-(22) also follow directly using the formulas (4)-(9). 


\section{REFERENCES}

Aigner, M. (1979). Combinatorial Theory, Berlin: SpringerVerlag.
Veljan, D. (2001). Kombinatorna i diskretna matematika [Combinatorial and Discrete Mathematics], Zagreb: Algoritam.

Vugdalić, R. (2009). The value of certain combinatorics sum. Journal of Mathematics, Vol.5 (18), 247-255. 